\title{
Heat transfer in rotating Rayleigh-Bénard convection with rough plates
}

\author{
Pranav Joshi $^{1,2, \dagger}$ Hadi Rajaei $^{1}$, Rudie P. J. Kunnen ${ }^{1}$ \\ and Herman J. H. Clercx ${ }^{1}$ \\ ${ }^{1}$ Fluid Dynamics Laboratory, Department of Applied Physics and J. M. Burgers Center \\ for Fluid Dynamics, Eindhoven University of Technology, P.O. Box 513, \\ $5600 \mathrm{MB}$ Eindhoven, The Netherlands \\ ${ }^{2}$ Experimental Aerodynamics Division, National Aerospace Laboratories, Bangalore 560017, India
}

(Received 9 July 2017; revised 4 September 2017; accepted 11 September 2017;

first published online 3 October 2017)

This experimental study focuses on the effect of horizontal boundaries with pyramid-shaped roughness elements on the heat transfer in rotating Rayleigh-Bénard convection. It is shown that the Ekman pumping mechanism, which is responsible for the heat transfer enhancement under rotation in the case of smooth top and bottom surfaces, is unaffected by the roughness as long as the Ekman layer thickness $\delta_{E}$ is significantly larger than the roughness height $k$. As the rotation rate increases, and thus $\delta_{E}$ decreases, the roughness elements penetrate the radially inward flow in the interior of the Ekman boundary layer that feeds the columnar Ekman vortices. This perturbation generates additional thermal disturbances which are found to increase the heat transfer efficiency even further. However, when $\delta_{E} \approx k$, the Ekman boundary layer is strongly perturbed by the roughness elements and the Ekman pumping mechanism is suppressed. The results suggest that the Ekman pumping is re-established for $\delta_{E} \ll k$ as the faces of the pyramidal roughness elements then act locally as a sloping boundary on which an Ekman layer can be formed.

Key words: Bénard convection, geophysical and geological flows, rotating turbulence

\section{Introduction}

Rayleigh-Bénard convection (RBC), which is a representative system to study buoyancy driven flows, is the flow driven by the temperature difference, $\Delta T$, between a heated horizontal plate and a parallel cooled plate a distance $H$ above it. In nature, such flows invariably occur on rough boundaries, e.g. mountainous terrain for atmospheric flows and the undulating bed in the case of oceanic processes. Even in technological applications, a surface can behave as a rough wall when the boundary

$†$ Email address for correspondence: pranav.rk.joshi@gmail.com 


\section{P. Joshi, H. Rajaei, R. P. J. Kunnen and H. J. H. Clercx}

layer on it becomes thin at sufficiently strong buoyancy forcing. On the other hand, the effects of rotation can be prominent at geophysical scales, e.g. planetary interiors (Aurnou et al. 2015), as well as in applications like chemical vapour deposition used to manufacture semiconductor chips. Although numerous researchers have studied the effects of rotation and of rough horizontal walls on RBC separately, leading to a better understanding of such flows, studies on the combined effects of rotation and roughness have been absent. The present work addresses this gap in our understanding by studying the impact of rough heat transfer walls on rotating RBC.

The strength of the buoyancy forcing in $\mathrm{RBC}$ is characterized by the Rayleigh number $R a=g \alpha \Delta T H^{3} /(\nu \kappa)$, where $\alpha, v$ and $\kappa$ are the isobaric thermal expansion coefficient, kinematic viscosity and thermal diffusivity of the fluid and $g$ is the gravitational acceleration. Other parameters that affect the system response are the Prandtl number $\mathrm{Pr}=v / \kappa$, the aspect ratio $\Gamma=D / H$ and the Rossby number $R o=\sqrt{g \alpha \Delta T / H} /(2 \Omega)$, where $\Omega$ is the angular velocity of the RBC system about an axis normal to the plates and $D$ is the lateral dimension of the system, which, for a cylindrical geometry, is the diameter. The response of the RBC system is typically expressed in terms of the Nusselt number $N u=q H /(\Delta T \lambda)$, where $q$ is the heat flux density and $\lambda$ is the thermal conductivity of the fluid. At $R a \sim 10^{9}$ and $\Gamma=1$, relevant to the present study, the flow in the absence of rotation is characterized by the thermal plumes emitted from the top and bottom walls. These plumes give rise to a domain-filling closed flow structure, the large-scale circulation (LSC), which in turn convects the plumes from one wall to the other (e.g. see Xi, Lam \& Xia 2004). The heat transfer in this regime is well described by the Grossmann-Lohse (GL) theory (Grossmann \& Lohse 2000, 2001, 2002), and the Ra-dependence of $N u$ can be approximated by $N u \sim R a^{\gamma}$, with $\gamma \approx 0.3$ (Funfschilling et al. 2005). For an overview of recent developments in RBC, see, e.g., Ahlers, Grossmann \& Lohse (2009).

The flow structure and $N u$ remain largely unaffected by the Coriolis force under weak rotation, i.e. when $R o \gtrsim R o_{c}$ (regime I). For $\Gamma=1, R o_{c} \approx 2.5$ (Kunnen, Clercx \& Geurts 2008; Stevens et al. 2009; Weiss et al. 2010). For $\operatorname{Pr} \sim O(10)$, as $R o$ decreases below $R o_{c}$, the LSC breaks down and the thermal plumes take the form of vertical columns of hot/cold fluid with predominantly axial vorticity emanating from the bottom/top walls. These vortical columnar plumes, which form as a result of the intensification of the cyclonic vorticity in the fluid converging into the emerging plumes, propagate deeper into the bulk flow as $R o$ is decreased below $R o_{c}$ (regime II), see, e.g., Kunnen, Clercx \& Geurts $(2010 a)$. This growth in the vertical extent of the columnar vortices is a result of the Taylor-Proudman-like phenomenon, in which axial variations in the flow are suppressed by rotation, an effect that grows stronger as Ro decreases. These columns draw more fluid from the Ekman boundary layers (the frictional layers on the horizontal walls in rotating flows) (Pedlosky 1987) as the radial pressure gradients associated with them are superposed on the Ekman layers. This phenomenon, commonly referred to as Ekman pumping (Rossby 1969; Pedlosky 1987; Vorobieff \& Ecke 2002), leads to a steady increase in $N u$ until $R o \approx 0.1$ (for $\operatorname{Ra} \sim 10^{9}, \operatorname{Pr} \sim 6$ and $\Gamma=1$, relevant to the present study), when the vortical columns span a substantial fraction of the RBC cell height. Since rotation suppresses the Rayleigh-Bénard instability (Chandrasekhar 1961; Rossby 1969), a further decrease in $R o$ into regime III is characterized by a damping of the turbulence and a decrease in $N u$ (Rossby 1969; Kunnen, Clercx \& Geurts 2006; King et al. 2009; Stevens et al. 2009; Kunnen, Geurts \& Clercx 2010b; Julien et al. 2012; Cheng et al. 2015).

The impact of the wall roughness on the heat transfer in non-rotating RBC is largely determined by the height of the roughness elements, $k$, in relation to the thermal and 


\section{Rotating convection with rough plates}

viscous boundary layer thicknesses, $\delta_{T}$ and $\delta_{v}$ respectively. However, most previous studies of convection over rough walls have been performed for $\operatorname{Pr} \sim O(1-10)$, for which $\delta_{T}$ and $\delta_{v}$ are of the same order. Perhaps hence, these studies have mostly focused on the interaction of the roughness with the thermal boundary layer, the mean thickness of which is easily estimated as $\delta_{T} \approx H / 2 N u$. Below a critical $R a$, the thermal boundary layer is thicker than $k$, and $N u$ for the rough surface, $N u_{R}$, is similar to that for a smooth wall, $N u_{S}$. As shown by Tisserand et al. (2011), $N u_{R}$ can also be slightly lower than $N u_{S}$ as the fluid inside the troughs of the roughness is near-stagnant, which results in a locally thick thermal boundary layer. As $R a$ increases and $\delta_{T}$ becomes smaller than $k$, the roughness elements protrude out of the thermal boundary layer and increase the emission of plumes (Du \& Tong 2000; Stringano, Pascazio \& Verzicco 2006), and thus the heat transfer in comparison with smooth walls. For large $\operatorname{Pr}\left(\delta_{v} \gg\right.$ $\delta_{T}$ ), Xie \& Xia (2017) show that $\delta_{v}$ also becomes smaller than $k$ at sufficiently high $R a$, and thus both the thermal and the viscous boundary layers are strongly perturbed by the rough wall.

Although there exists a consensus on the effect of roughness on the magnitude of $N u$, its effect on $\gamma$ seems to be unclear. Some early studies (Shen, Tong \& Xia 1996; Du \& Tong 1998; Ciliberto \& Laroche 1999) report no change in $\gamma$ from its value for smooth plates when $\delta_{T}<k$, although $N u_{R}$ can be larger than $N u_{S}$ by up to $80 \%$. However, later studies, e.g. Qiu, Xia \& Tong (2005) and Stringano et al. (2006), have measured $\gamma$ in the range $0.35-0.37$. They ascribe the lower $\gamma$ observed in the earlier studies to the poor thermal diffusivity of the plate material (brass) used in them, which exacerbates the inherently non-isothermal character of a rough plate in an experiment. However, calculating $N u$ for individual plates at $R a \sim O\left(10^{12}\right)$, Tisserand et al. (2011) measure $\gamma$ as high as 0.5 for a rough aluminium plate, which has thermal properties poorer than those of a similar copper plate. The Nusselt number for an individual plate is calculated as $N u_{p}=q H /\left(2\left|T_{p}-T_{c}\right| \lambda\right)$ (Tisserand et al. 2011; Wei et al. 2014), where $T_{p}$ and $T_{c}$ are the temperatures of the plate and the RBC cell centre respectively. Wei et al. (2014) find that, based on the boundary conditions, i.e. constant temperature or constant heat flux, and the nature of the opposite plate, $\gamma$ for a rough plate can vary between 0.29 and 0.39 . However, with both plates rough, they observe $\gamma=0.35$ for the global $\mathrm{Nu}$. Xie \& Xia (2017) show that $\gamma$ is higher in the regime $\delta_{T}<k<\delta_{v}$ than in the regime $k>\delta_{v}$ and, in qualitative agreement with the 2D simulations of Toppaladoddi, Succi \& Wettlaufer (2017), that $\gamma$ also depends on the aspect ratio of the roughness elements.

The columnar vortices, which are presumed to be responsible for increasing the heat transfer under rotation, are intricately linked with the dynamics of the Ekman boundary layer on the horizontal surfaces. Since roughness modifies the boundary layer structure in non-rotating convection, some natural questions to ask are whether and how it affects the Ekman vortices under rotation. Although there have been prior investigations studying geostrophic turbulence (LaCasce \& Brink 2000) and stratified Ekman layers (see, e.g., Garrett, MacCready \& Rhines 1993) on sloping boundaries, the impact of roughness on the Ekman layer and the Ekman pumping mechanism in the context of rotating natural convection has not been explored. The interaction of the effects of rotation and roughness can be important in geophysical flows. Even in technological applications, roughness effects can become relevant as the Ekman boundary layer becomes thin under strong rotation. In this study, we introduce pyramid-shaped roughness elements on the top and/or bottom walls and measure the heat transfer as a function of the rotation rate. In the next section, we describe the experimental apparatus and measurement procedures. In $\S \S 3$ and 4 , we present 


\section{P. Joshi, H. Rajaei, R. P. J. Kunnen and H. J. H. Clercx}
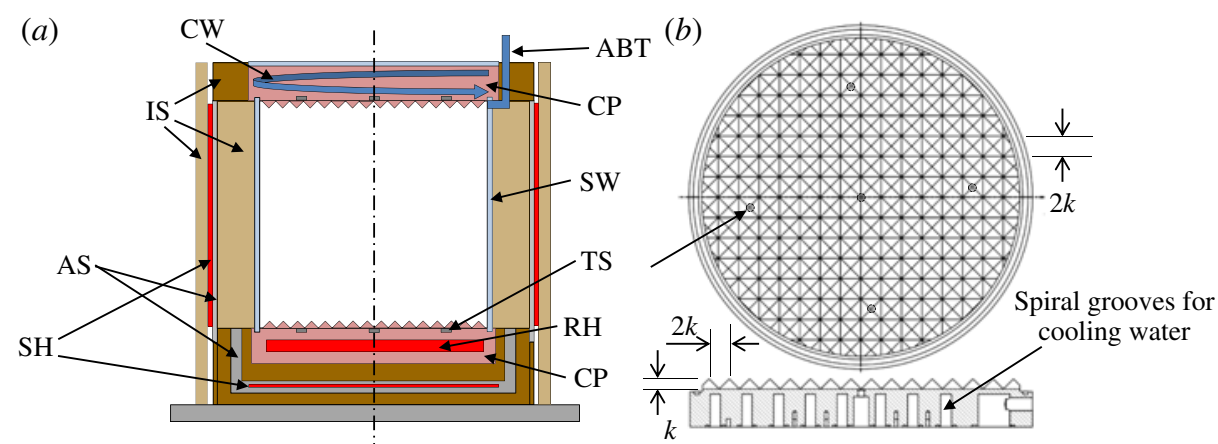

FIGURE 1. (a) Schematic of the experimental set-up: SW, cylindrical Plexiglass sidewall; $\mathrm{CP}$, top and bottom copper plates; $\mathrm{RH}$, heating resistors fitted into the bottom copper plate; $\mathrm{CW}$, cooling water recirculating through the top copper plate; ABT, air-bleed tube which is connected to an overhead flask; SH, secondary heaters; AS, aluminium adiabatic shields; IS, insulating shields; TS, temperature sensors. The figure is not to scale. $(b)$ Details of the roughness geometry. The figure shows the top rough plate with $k=8 \mathrm{~mm}$.

the results and show that, depending on the size of the roughness elements relative to the Ekman boundary layer thickness, rotation can either enhance or suppress the heat transfer in relation to that under non-rotating conditions.

\section{Experimental set-up and measurement procedures}

Figure 1 shows a schematic of the $\Gamma \approx 1$ cylindrical Rayleigh-Bénard cell used in this study. Only the important features of this cell are described here. For details see Kunnen et al. (2011) and Joshi et al. (2016). The heat transfer plates with $D=$ $250 \mathrm{~mm}$ are made out of solid copper blocks. Triangular grooves are machined on the surfaces of these plates, producing a square array of pyramids with height $k$ and a square base of side $2 k$ (see figure $1 b$ ). The plates are $30 \mathrm{~mm}$ thick (at the base of the pyramids for rough plates). Two roughness sizes, $k=1.5 \mathrm{~mm}$ and $8 \mathrm{~mm}$, have been used. The equivalent location of the rough surface is fixed at the mid-height of the pyramids, determining the effective height $H_{\text {eff }}$ and the effective aspect ratio $\Gamma_{\text {eff }}$ of the RBC cell in each case. Table 1 lists the five combinations of the rough and smooth plates used, as well as $H_{\text {eff }}$ and $\Gamma_{\text {eff }}$ for each case. Water is used as the working fluid. Heat is supplied to the bottom plates using ohmic heating and the top plates are cooled by recirculating water through them. A constant-temperature boundary condition is imposed at both the top and bottom plates, with temperatures $T_{t}$ and $T_{b}$ respectively. Five thermistors are provided in each of the plates, $0.7 \mathrm{~mm}$ from the wetted surface for the smooth plates and $1.0 \mathrm{~mm}$ from the base of the pyramids for the rough plates. The thermistors are calibrated with logarithmic polynomial fits and measure the temperature with an accuracy of $\pm 0.01{ }^{\circ} \mathrm{C}$. The RBC cell is mounted on a table, the rotation rate of which can be controlled from $\Omega=0$ to $10 \mathrm{rad} \mathrm{s}^{-1}$ with a resolution of $0.001 \mathrm{rad} \mathrm{s}^{-1}$ and a relative accuracy of $0.5 \%$. The maximum rotation rate used in the present study is $\Omega=2.5 \mathrm{rad} \mathrm{s}^{-1}$, yielding a Froude number of $F r=\Omega^{2}(D / 2) / g=0.079$, i.e. centrifugal effects are minor. For more details on the experimental set-up, we refer the reader to Kunnen et al. (2011).

Data recorded at a sampling frequency of $1 \mathrm{~Hz}$ under steady state for longer than $6 \mathrm{~h}$ are used to calculate the mean quantities. The temperatures measured by the five 
Rotating convection with rough plates
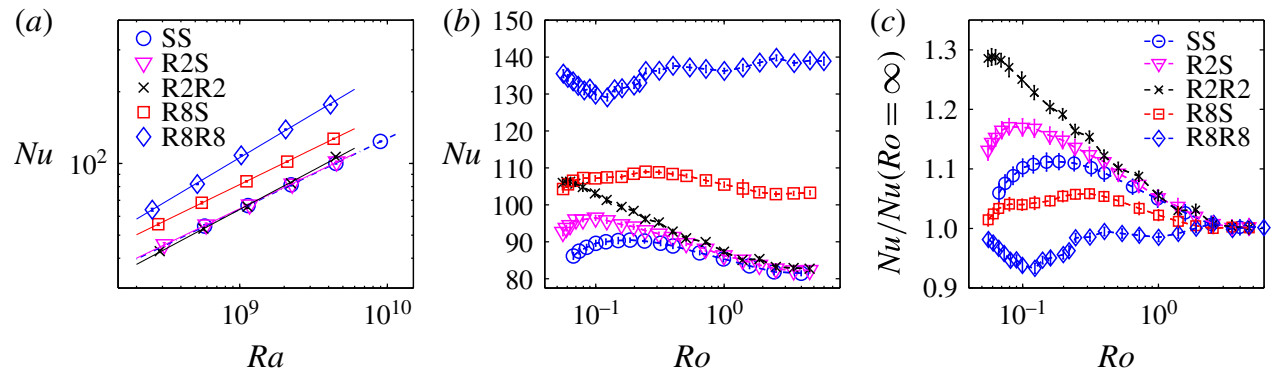

FIgURE 2. Variation of (a) $N u$ with $R a$ for $R o=\infty$, (b) $N u$ with $R o$ and (c) $N u / N u(R o=$ $\infty)$ with $R o$ for all $k$ at $R a=2.2 \times 10^{9}$. The nomenclature of the plate combinations is given in table 1 . In $(a)$, the straight lines represent the power law fits to the data, which result in different values of the exponent $\gamma$ : dashed-dotted blue line, SS, $\gamma=0.298$; solid magenta line, R2S, $\gamma=0.297$; solid black line, R2R2, $\gamma=0.330$; solid red line, R8S, $\gamma=0.302$; solid blue line, R8R8, $\gamma=0.369$.

$\begin{array}{lccccc}\text { Case } & \text { Top plate } & \text { Bottom plate } & H_{\text {eff }}(\mathrm{mm}) & \Gamma_{\text {eff }}=D / H_{\text {eff }} & \gamma \\ \text { SS } & \text { Smooth } & \text { Smooth } & 250.0 & 1.0 & 0.298 \pm 0.002 \\ \text { R2S } & \text { Rough, } k=1.5 \mathrm{~mm} & \text { Smooth } & 249.25 & 1.003 & 0.297 \pm 0.006 \\ \text { R2R2 } & \text { Rough, } k=1.5 \mathrm{~mm} & \text { Rough, } k=1.5 \mathrm{~mm} & 248.5 & 1.006 & 0.330 \pm 0.013 \\ \text { R8S } & \text { Rough, } k=8 \mathrm{~mm} & \text { Smooth } & 246.0 & 1.0163 & 0.302 \pm 0.002 \\ \text { R8R8 } & \text { Rough, } k=8 \mathrm{~mm} & \text { Rough, } k=8 \mathrm{~mm} & 242.0 & 1.0331 & 0.369 \pm 0.012\end{array}$

TABLE 1. The combinations of rough and smooth plates used in the present study. The nomenclature of the various cases uses $\mathrm{S}$ for smooth, $\mathrm{R}$ for rough and a number denoting the height of the roughness element, $k$. For example, R8S denotes an experiment using a rough top plate with $k=8 \mathrm{~mm}$ and a smooth bottom plate. The values of $\gamma$ in the relation $N u \sim R a^{\gamma}$ are estimated using least-squares fits to the data. The uncertainty in $\gamma$ is expressed as $\gamma \pm 2 \Delta_{\gamma}$, where $\Delta_{\gamma}$ is the standard error in the estimate of $\gamma, \Delta_{\gamma}^{2}=$ $\left(s_{\phi \phi} s_{\psi \psi}-s_{\phi \psi}^{2}\right) /\left[(n-2) s_{\phi \phi}^{2}\right], n$ is the number of data points $(R a, N u)$ used for the linear regression, $\phi=\ln (R a), \psi=\ln (N u)$ and $s_{\phi \psi}=\sum_{i=1}^{n}\left(\phi_{i}-\bar{\phi}\right)\left(\psi_{i}-\bar{\psi}\right)$ is the covariance of $\phi$ and $\psi$ (Acton 1966). An overbar denotes the sample mean for a data set.

sensors in the top (bottom) plate are averaged to calculate $T_{t}\left(T_{b}\right)$. Fluid properties are evaluated at $T_{\text {mean }}=\left(T_{t}+T_{b}\right) / 2=24.2 \pm 0.3{ }^{\circ} \mathrm{C}(\operatorname{Pr}=6.2)$. Heat lost from the bottom plate to the surroundings, which is less than $4 \%$ of the input heat flux $Q_{I}$ at $R a=3 \times 10^{8}$ and decreases to $0.2 \%$ of $Q_{I}$ at $R a=4.5 \times 10^{9}$, is subtracted from $Q_{I}$ to obtain the net heat flux density $q$.

\section{Results}

\subsection{Effect of rough plates on heat transfer without rotation}

Figure 2(a) shows the variation of $N u$ with $R a$. The length of the error bars in figure 2 represents $4 \Delta_{\phi}$, where $\Delta_{\phi}=\sigma_{\phi} / \sqrt{\left(t_{0} / \tau_{0}\right)}$ is the standard error of the mean value $\langle\phi\rangle$ of the concerned variable $\phi$. Here, $\sigma_{\phi}$ is the standard deviation of $\phi, t_{0}$ is the length of the time series for $\phi(t)$ used to calculate $\langle\phi\rangle, t$ is time and $\tau_{0}$ is the time interval for the temporal autocorrelation of $\phi, C_{\phi}(\tau)=\langle\phi(t) \phi(t+\tau)\rangle / \sigma_{\phi}^{2}$, to decay to $1 / e$. 


\section{P. Joshi, H. Rajaei, R. P. J. Kunnen and H. J. H. Clercx}

It should be noted that in many cases the error bars are smaller than the size of the symbols.

In the following discussion, different plate combinations are indicated by the corresponding subscripts to the variable (for the nomenclature, see table 1 and the caption). It should be noted that the $R a$ range for the case $\mathrm{SS}, 6 \times 10^{8}-9 \times 10^{9}$, is different from that for all the cases with rough plates, approximately $3 \times 10^{8}-4.5 \times 10^{9}$. A least-squares fit to the data for the case SS yields $\gamma_{S S}=0.3$. Both $\gamma_{S S}$ and $N u_{S S}$ agree well with those reported in earlier studies using smooth plates (e.g. Funfschilling et al. 2005). The Nusselt number and $\gamma$ for the case R2S are indistinguishable from those for the case SS. However, when both plates are rough, $N u_{R 2 R 2}$ shows slight deviations (less than $5 \%$ ) from $N u_{S S}$. The exponent $\gamma_{R 2 R 2}$ is also higher, 0.33. For the cases $\mathrm{R} 2 \mathrm{~S}$ and R2R2, $\delta_{T} \approx H / 2 N u$ decreases from approximately $2.9 \mathrm{~mm}$ at $R a=2.9 \times 10^{8}$ to $1.2 \mathrm{~mm}$ at $R a=4.5 \times 10^{9}$. Although $\delta_{T}<k$ at the highest $R a$, we do not observe any substantial increase in $N u$ (less than $5 \%$ for the case R2R2) above the case SS, perhaps because $\delta_{T}$ is still only slightly smaller than $k$. Indeed, previous data with symmetrical RBC cells, e.g. from Shen et al. (1996), Stringano et al. (2006) and Wei et al. (2014), appear to indicate that the increased heat transfer is manifested only when $\delta_{T}$ becomes smaller than approximately $2 k / 3$.

For the cases R8S and R8R8, the thermal boundary layer thickness is always significantly smaller $\left(\delta_{T}<2.2 \mathrm{~mm}\right)$ than the height of the pyramid-like structures $(k=8 \mathrm{~mm})$. As a result, $N u$ for these cases is substantially higher than that for the case SS. As expected, the heat transfer when both plates are rough (R8R8) is higher than that with only one rough plate $(\mathrm{R} 8 \mathrm{~S})$. At $R a=4.1 \times 10^{9}, N u$ for the cases $\mathrm{R} 8 \mathrm{~S}$ and R8R8 is approximately $26 \%\left(k / \delta_{T} \approx 8\right)$ and $80 \%\left(k / \delta_{T} \approx 11\right)$ higher respectively than that for the case SS. The latter increase is comparable to the $76 \%$ reported by $\mathrm{Du} \&$ Tong (1998) for pyramidal roughness elements at similar $R a$ (their data suggest $\left.k / \delta_{T} \approx 9\right)$. However, unlike their exponent, which maintains the same value as for the smooth case, we observe $\gamma_{R 8 R 8} \approx 0.37$. This value is close to $\gamma=0.35$ measured for pyramidal roughness by Qiu et al. (2005) $\left(k / \delta_{T} \approx 1-16\right)$ and Wei et al. (2014) $\left(k / \delta_{T} \approx 0.8-2.5\right)$, and agrees with $\gamma=0.37$ obtained by Stringano et al. (2006) from their direct numerical simulations (DNS) with axisymmetric grooves $\left(k / \delta_{T} \approx 2-31\right)$. Stringano et al. (2006) suggest that the lower exponent in Du \& Tong (1998) is the result of an interplay between the enhanced emission of plumes due to the roughness and the decrease in heat transfer due to the non-ideal brass plates. It is also clear from the above comparison that $\gamma$ is independent of $k / \delta_{T}$. It should be noted that $\gamma_{R 8 S}$ (figure $2 a$ and table 1) is indistinguishable from $\gamma_{S S}=0.30$, in line with the observations of Wei et al. (2014) for a similar asymmetric configuration.

To summarize the above discussion, in line with previous studies for $\operatorname{Pr} \gtrsim 1$, we observe no enhancement in the heat transfer when $\delta_{T} \gtrsim k$. However, for $\delta_{T}$ smaller than $k / 4$, a significant increase in $N u$ is observed. The heat transfer enhancement with a doubly rough $\mathrm{RBC}$ cell is larger than that with an asymmetric cell. Although roughness increases $N u$ for sufficiently large $k / \delta_{T}, \gamma$ for the asymmetric cell is indistinguishable from that for the smooth cell, and only shows an increase when both walls are rough. For the present data, $\gamma$ is higher for larger $k$. This difference, however, may be a result of different $k / \delta_{v}$ for the cases R2R2 and R8R8. According to the GL theory (Grossmann \& Lohse 2001; Stevens et al. 2013b), for $\operatorname{Pr}=6.2, \delta_{v}$ over a smooth wall decreases from approximately $10 \mathrm{~mm}$ to $5 \mathrm{~mm}$ as $R a$ is increased from $2 \times 10^{8}$ to $5 \times 10^{9}$. Thus, the $k=8 \mathrm{~mm}$ roughness elements are expected to strongly perturb the viscous boundary layer, while the $k=1.5 \mathrm{~mm}$ roughness elements are likely to be buried deep within it. 


\section{Rotating convection with rough plates}

\subsection{Effect of rough plates on heat transfer under rotation}

Figure 2(b) shows the variation of $N u$ with $R o$ at $R a=2.2 \times 10^{9}$. When both plates are smooth, the behaviour of $N u$ agrees well with that reported in earlier studies (e.g. Kunnen et al. 2006; Zhong \& Ahlers 2010; Kunnen et al. 2011; Stevens, Clercx \& Lohse 2013a): an increase in $N u$ as $R o$ decreases below the critical value $R o_{c} \approx 2.5$ and a maximum attained at $R o \approx 0.2$, which is assumed to indicate the transition to regime III. It is evident from figure $2(b)$ that at all the rotation rates studied, $N u$ for $k=8 \mathrm{~mm}$ is substantially higher than that for smooth plates, while $N u$ for $k=1.5 \mathrm{~mm}$ differs significantly from $N u_{S S}$ only after $R o$ decreases below approximately 0.5 .

To better assess the above trends, the variation of $N u / N u(R o=\infty)$ with $R o$ at $R a=2.2 \times 10^{9}$ is shown in figure 2(c). Evidently, the value of $R o$ that marks the end of regime I with negligible effect of rotation, i.e. $R o_{c} \approx 2.5$, is unchanged in the presence of the rough plates. For $k=1.5 \mathrm{~mm}$ (data sets R2R2 and R2S), the trends for $\mathrm{Nu} / \mathrm{Nu}(\mathrm{Ro}=\infty)$ are qualitatively similar to those for the smooth plates, although some quantitative differences are observed. For smooth plates, the maximum value of $N u / N u(R o=\infty)$ is 1.11. This maximum is significantly higher in the presence of $k=1.5 \mathrm{~mm}$ roughness: 1.17 and 1.29 for the sets $\mathrm{R} 2 \mathrm{~S}$ and $\mathrm{R} 2 \mathrm{R} 2$ respectively. Furthermore, the maxima occur at lower Rossby numbers, $R o_{R 2 S} \approx 0.08$ and $R o_{R 2 R 2} \approx$ 0.06 , indicating that the regime II with enhanced heat transfer is more robust and the transition to regime III is delayed to higher rotation rates. The results for $k=8 \mathrm{~mm}$ are qualitatively as well as quantitatively different from those for smooth plates. For the case $\mathrm{R} 8 \mathrm{~S}$, the maximum value of $N u / N u(R o=\infty)$ at $R o \approx 0.3$ is 1.06 , which is significantly smaller than the value of 1.11 for the reference case SS. Furthermore, instead of a steady decay as $R o$ is decreased further, $N u / N u(R o=\infty)$ shows a weak plateau at $R o \approx 0.1$. However, the most significant deviations from the case SS are observed when both plates are rough. In contrast to all other cases, rotation decreases the heat transfer for the case R8R8: $N u / N u(R o=\infty)$ is always smaller than 1 for $R o \lesssim 2.5$. After a broad plateau around $R o \approx 0.4, N u / N u(R o=\infty)$ reaches a minimum at $R o \approx 0.12$, whereafter it increases as $R o$ is decreased further. Although one should expect the heat transfer to decay again at sufficiently high rotation rates, this trend of increase in $N u$ for $R o \lesssim 0.1$ is in marked contrast to the case SS, which exhibits a suppression of heat transfer. Thus, the flow regimes observed in the reference case SS appear to be greatly modified in the presence of the $k=8 \mathrm{~mm}$ roughness.

To recapitulate, although the variation of $N u$ with $R o$ remains qualitatively unchanged for $k=1.5 \mathrm{~mm}$, the maximum of $N u / N u(R o=\infty)$ is significantly larger than that for the case SS, and regime II extends to lower Ro. For the asymmetric $k=8 \mathrm{~mm}$ case (R8S), the enhancement in $N u$ under rotation is significantly lower than that for SS, while for the symmetric case R8R8, rotation decreases $N u$ in comparison to its value at $R o=\infty$. In addition to the lower magnitudes, $N u / N u(R o=\infty)$ for $k=8 \mathrm{~mm}$ shows marked qualitative differences in comparison to the case SS, indicating the possibility of a different underlying flow structure.

\section{Discussion}

It is evident from $\S 3$ that the effect of rotation on the heat transfer depends critically on the roughness size. It should be noted that for both $k$ values, the trends for the rough-smooth combinations (R2S and R8S) are intermediate to those for the reference case SS and the respective rough-rough combinations (R2R2 and R8R8). Since we do not measure the temperature at the RBC cell centre, and the sidewall temperature may not be a good approximation to the bulk temperature at low $R o$ 
(a)

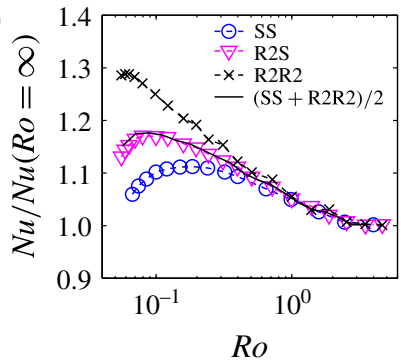

(b)

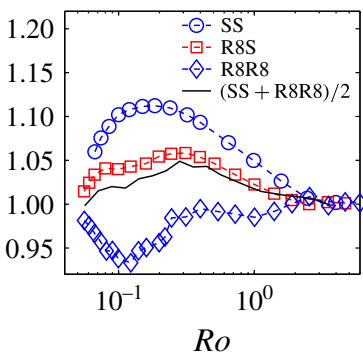

(c)

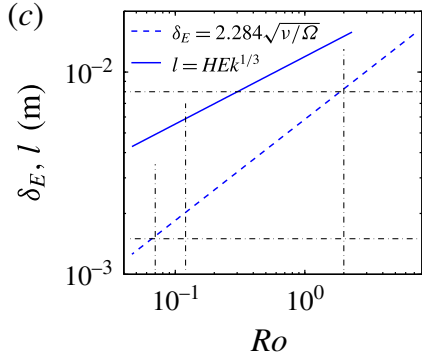

FIgURE 3. Variation of $N u / N u(R o=\infty)$ with $R o$ at $R a=2.2 \times 10^{9}$ for $(a) k=1.5 \mathrm{~mm}$ and $(b) k=8 \mathrm{~mm}$. The variation of the Ekman boundary layer thickness $\delta_{E}$ over a smooth wall and the characteristic horizontal length scale of the vortical columns $l=H E k^{1 / 3}$ (King, Stellmach \& Buffett 2013) with Ro is shown in (c). In $(a)$ and $(b)$, the black solid lines represent the average of the data for the cases SS, and R2R2 and R8R8 respectively. In $(c)$, the $R o$ values for all the cases differ negligibly (less than $2 \%$ ) from each other, $R o_{S S} \approx$ $R o_{R 2 R 2} \approx R o_{R 8 R 8}$, since $H_{\text {eff }} \approx 250 \mathrm{~mm}$ for all these cases (see table 1). The vertical black dash-dotted lines denoting $R o=0.07,0.12$ and 2.0, and the horizontal black dash-dotted lines denoting the lengths $8 \mathrm{~mm}$ and $1.5 \mathrm{~mm}$ are provided to aid visualization.

(Kunnen et al. 2011), we cannot estimate $N u$ for the plates separately. However, to indicate the broad trends, we present the mean of $N u / N u(R o=\infty)$ for the cases SS and R2R2 in figure 3(a) and that for the cases SS and R8R8 in figure 3(b). The averages of the data for the symmetric smooth and rough cases approximate very well, both qualitatively and quantitatively, the data for the corresponding asymmetric configurations. Although the agreement in the case of $k=8 \mathrm{~mm}$ is not as good as for $k=1.5 \mathrm{~mm}$, the overall trend for the case R8S, i.e. a non-monotonic decrease in $N u$ with decreasing $R o$ for $0.055 \lesssim R o \lesssim 0.3$, is very well reproduced by the average of $\mathrm{Nu}$ for the cases SS and R8R8. This agreement suggests that in a rotating asymmetric RBC cell, each plate maintains its heat transfer characteristics, and the trend for the global $N u$ is a combination of those for $N u$ for the individual plates.

As discussed earlier, the heat transfer primarily depends on the interaction of the roughness with the thermal and viscous boundary layers. At $R o \approx 2.5$, the Prandtl-Blasius viscous boundary layer undergoes a transition to the Ekman type (Stevens et al. 2009; Rajaei et al. 2016). Similarly to $N u_{S S}, N u_{R 2 R 2}$ increases when $R o$ is decreased below 2.5 (figure $3 a$ ) as the Ekman pumping mechanism starts to draw hot/cold fluid from the bottom/top thermal boundary layers. Figure 3(c) shows the variation of the Ekman boundary layer thickness $\delta_{E}=2.284 \sqrt{v / \Omega}$ (Rajaei et al. 2016) with $R o\left(R o \approx R o_{R 2 R 2} \approx R o_{R 8 R 8}\right)$ over a smooth wall. In the vicinity of the transition at $R o \approx 2.5, \delta_{E} \approx 9 \mathrm{~mm}$ is significantly thicker than $k=1.5 \mathrm{~mm}$. Thus, the roughness elements are buried deep inside the Ekman layer and their effect on the heat transfer is not evident. The effect of roughness becomes significant only for $R o \lesssim 0.5$ (figure $3 a$ ) when $\delta_{E}$ decreases to approximately $4 \mathrm{~mm}$ (figure $3 c$ ). It should be noted that the Ekman vortices draw fluid primarily from the bulk of the boundary layer rather than the near-wall region (Stevens, Clercx \& Lohse 2010). Thus, it is only when $\delta_{E} / k$ becomes small enough that the roughness elements get exposed to a sufficiently strong radial inflow of the boundary layer fluid into the Ekman vortices. It should be noted that, as discussed in $\S 3.1, \delta_{T} \approx k$ for $R o=\infty$ at $R a=2.2 \times 10^{9}$. Although $\delta_{T}$ can increase substantially in regime III compared with regimes I and II (Kunnen et al. 2006, 2010b, 2016), it is expected to remain largely 


\section{Rotating convection with rough plates}

unchanged and reasonably well estimated by $\delta_{T} \approx H / 2 N u$ in regime II (Kunnen et al. $2006,2010 b$ ). Since $\delta_{T} \approx k$, the radial inflow over the roughness elements is likely to generate more thermal structures from the perturbed thermal boundary layer. These structures enter the Ekman vortices and increase their heat transfer efficiency, which is reflected in the higher $N u / N u(R o=\infty)$ for the case R2R2 in comparison with the case SS. For smooth walls, the increase in the heat transfer due to Ekman pumping is small or absent when $\operatorname{Pr} \gg 1$ or $\operatorname{Pr} \ll 1$ (Stevens et al. 2010). However, for the case R2R2, an increase in $N u / N u(R o=\infty)$ in comparison with the case SS can also be expected when $\delta_{T} \ll k(\operatorname{Pr} \gg 1$ or high $R a)$ due to a radial-flow-induced enhanced emission of hot/cold plumes from the thermal boundary layer at the tips of the roughness elements. On the other hand, when $\delta_{T} \gg k(P r \ll 1$ or low $R a)$, the enhanced emission of structures from the roughness elements may not be as effective in increasing the heat transfer efficiency due to the high thermal diffusivity of the fluid. The discussed interaction of the near-wall flow with the roughness for the case R2R2 to generate higher thermal/momentum fluctuations could be qualitatively similar to that observed in turbulent boundary layers over rough walls (Jimenez 2004) and the interaction of the LSC with rough walls in non-rotating RBC (Wagner \& Shishkina 2015).

The transition from regime II to regime III, indicated by the maximum of $\mathrm{Nu}$, is delayed to lower $R o$ in the presence of the $k=1.5 \mathrm{~mm}$ roughness (figure $3 a$ ). For smooth walls, it is known that this transition is accompanied by an increased damping of turbulence by rotation (Boubnov \& Golitsyn 1990; Joshi et al. 2016). Since a rough wall is expected to lead to higher turbulent fluctuations, it is not surprising that this transition is delayed to smaller $R o$ in the presence of rough walls. However, it should be noted that although $\delta_{E}$ remains larger than $k=1.5 \mathrm{~mm}$ for moderate rotation rates, it becomes equal to $k$ at $R o \approx 0.07$. Consequently, the Ekman boundary layer is strongly perturbed by the roughness, and this may lead to disruption of the Ekman pumping mechanism. This disruption explains the location $R o \approx 0.07$ of the end of regime II, after which $N u$ decays as Ro continues to decrease. If this is indeed the case, then the nature of the Ekman boundary layer in the vicinity of the transition, i.e. when $\delta_{E} \approx k$, is likely to be different from its well-known structure over smooth walls. However, at sufficiently low $R o$, when $\delta_{E} \ll k$, the faces of the pyramidal roughness elements may act like sloping boundaries. Consequently, the Ekman vortices may reappear, albeit with a slightly thicker Ekman layer $\delta_{E, \beta}=\delta_{E} \sqrt{\sec \beta}$ (Pedlosky 1987), where $\beta$ is the angle the slope makes with the horizontal. In the present case, $\delta_{E, \beta}=1.19 \delta_{E}$ for $\beta=45^{\circ}$. Unfortunately, the present experimental set-up cannot be operated at higher rotation rates to explore the low-Ro regime III for $k=1.5 \mathrm{~mm}$.

The disruption of the Ekman pumping mechanism when $\delta_{E} \approx k$ and its possible re-establishment when $\delta_{E} \ll k$ are also suggested by the data for the case R8R8. For this case, $\delta_{E}$ is already equal to $k$ at $R o \approx 2$ (figure $3 c$ ). Consequently, there is no heat transfer enhancement due to Ekman pumping. This, perhaps combined with the turbulence suppression under rotation, leads to a slight decrease in $N u$, approximately $2 \%$, for $R o \lesssim 2$. The Nusselt number continues to decrease as $R o$ decreases, possibly due to further suppression of turbulence. It reaches a minimum at $R o \approx 0.12$ and increases again as $R o$ is reduced further. It is possible that $\delta_{E} \approx k / 4$ at $R o=0.12$ is small enough in comparison with $k$ for re-establishment of the Ekman pumping mechanism. The characteristic horizontal length scale of the vortical convection columns, $l \sim H E k^{1 / 3}$ (King et al. 2013), where $E k=v / \Omega H^{2}$ is the Ekman number, is also shown in figure $3(c)$. At $R o=0.12, l \approx 6 \mathrm{~mm}$, i.e. slightly smaller than $k=8 \mathrm{~mm}$, which is also likely to aid this transition. However, further studies would be needed 


\section{P. Joshi, H. Rajaei, R. P. J. Kunnen and H. J. H. Clercx}

to provide strong evidence for this disruption and re-establishment of the Ekman layer. It should be noted that $N u$ is expected to decay again at sufficiently high rotation rates as the turbulence damping overwhelms the effect of the re-established Ekman pumping.

\section{Conclusion}

Heat transfer measurements have been performed in rotating $\mathrm{RBC}$ at $R a=2.2 \times 10^{9}$ and $\operatorname{Pr}=6.2$ in the presence of pyramid-shaped roughness elements over the horizontal plates. Our measurements in the absence of rotation agree well with those reported by other researchers. We observe that the effect of rotation on the heat transfer depends on the roughness size relative to the Ekman boundary layer thickness. The Ekman pumping mechanism remains largely unaffected by the roughness when $\delta_{E} \gg k$, as the roughness elements are buried deep inside the boundary layer. As $\delta_{E}$ becomes smaller, the roughness elements are exposed to the radial inflow of the fluid converging into the Ekman vortices. We believe that this interaction generates stronger momentum and temperature fluctuations, and hence leads to an increase in the heat transfer. However, when $\delta_{E} \approx k$, the Ekman boundary layer is strongly perturbed by the roughness and the Ekman pumping mechanism is disrupted. It is perhaps re-established when $\delta_{E} \ll k$, as the boundary layer flow may then be similar to that of an Ekman layer over a sloping boundary.

\section{Acknowledgement}

This work is supported by the Foundation for Fundamental Research on Matter (FOM), Netherlands.

\section{References}

ACton, F. S. 1966 Analysis of Straight-Line Data. Dover.

Ahlers, G., Grossmann, S. \& LohSE, D. 2009 Heat transfer and large scale dynamics in turbulent Rayleigh-Bénard convection. Rev. Mod. Phys. 81 (2), 503-537.

Aurnou, J. M., Calkins, M. A., Cheng, J. S., Julien, K., King, E. M., Nieves, D., Soderlund, K. M. \& STEllmach, S. 2015 Rotating convective turbulence in Earth and planetary cores. Phys. Earth Planet. Inter. 246, 52-71.

Boubnov, B. M. \& Golitsyn, G. S. 1990 Temperature and velocity field regimes of convective motions in a rotating plane fluid layer. J. Fluid Mech. 219, 215-239.

Chandrasekhar, S. 1961 Hydrodynamic and Hydromagnetic Stability. Oxford University Press.

Cheng, J. S., Stellmach, S., Ribeiro, A., Grannan, A., King, E. M. \& Aurnou, J. M. 2015 Laboratory-numerical models of rapidly rotating convection in planetary cores. Geophys. $J$. Intl 201, 1-17.

Ciliberto, S. \& LARoche, C. 1999 Random roughness of boundary increases the turbulent convection scaling exponent. Phys. Rev. Lett. 82 (20), 3998-4001.

DU, Y.-B. \& ToNG, P. 1998 Enhanced heat transport in turbulent convection over a rough surface. Phys. Rev. Lett. 81 (5), 987-990.

Du, Y.-B. \& Tong, P. 2000 Turbulent thermal convection in a cell with ordered rough boundaries. J. Fluid Mech. 407, 57-84.

Funfschilling, D., Brown, E., Nikolaenko, A. \& Ahlers, G. 2005 Heat transport by turbulent Rayleigh-Bénard convection in cylindrical samples with aspect ratio one and larger. J. Fluid Mech. 536, 145-154.

Garrett, C., MacCready, P. \& Rhines, P. 1993 Boundary mixing and arrested Ekman layers: rotating stratified flow near a sloping boundary. Annu. Rev. Fluid Mech. 25 (1), 291-323.

Grossmann, S. \& LohSE, D. 2000 Scaling in thermal convection: a unifying theory. J. Fluid Mech. 407, 27-56. 


\section{Rotating convection with rough plates}

Grossmann, S. \& Lohse, D. 2001 Thermal convection for large Prandtl numbers. Phys. Rev. Lett. 86 (15), 3316-3319.

Grossmann, S. \& Lohse, D. 2002 Prandtl and Rayleigh number dependence of the Reynolds number in turbulent thermal convection. Phys. Rev. E 66, 016305.

JimeneZ, J. 2004 Turbulent flows over rough walls. Annu. Rev. Fluid Mech. 36, 173-196.

Joshi, P., Rajaei, H., Kunnen, R. P. J. \& Clercx, H. J. H. 2016 Effect of particle injection on heat transfer in rotating Rayleigh-Bénard convection. Phys. Rev. Fluids 1 (8), 084301.

Julien, K., Knobloch, E., Rubio, A. M. \& Vasil, G. M. 2012 Heat transport in low-Rossbynumber Rayleigh-Bénard convection. Phys. Rev. Lett. 109 (25), 254503.

King, E. M., Stellmach, S.\& Buffett, B. 2013 Scaling behaviour in Rayleigh-Bénard convection with and without rotation. J. Fluid Mech. 717, 449-471.

King, E. M., Stellmach, S., Noir, J., Hansen, U. \& Aurnou, J. M. 2009 Boundary layer control of rotating convection systems. Nature 457 (7227), 301-304.

Kunnen, R. P. J., Clercx, H. J. H. \& Geurts, B. J. 2006 Heat flux intensification by vortical flow localization in rotating convection. Phys. Rev. E 74 (5), 056306.

Kunnen, R. P. J., Clercx, H. J. H. \& Geurts, B. J. 2008 Breakdown of large-scale circulation in turbulent rotating convection. Europhys. Lett. 84 (2), 24001.

Kunnen, R. P. J., Clercx, H. J. H. \& Geurts, B. J. $2010 a$ Vortex statistics in turbulent rotating convection. Phys. Rev. E 82 (3), 036306.

Kunnen, R. P. J., Geurts, B. J. \& Clercx, H. J. H. $2010 b$ Experimental and numerical investigation of turbulent convection in a rotating cylinder. J. Fluid Mech. 642, 445-476.

Kunnen, R. P. J., Ostilla-Mónico, R., van der Poel, E. P., Verzicco, R. \& Lohse, D. 2016 Transition to geostrophic convection: the role of the boundary conditions. J. Fluid Mech. 799, 413-432.

Kunnen, R. P. J., Stevens, R. J. A. M., Overkamp, J., Sun, C., van Heijst, G. J. F. \& ClerCX, H. J. H. 2011 The role of Stewartson and Ekman layers in turbulent rotating Rayleigh-Bénard convection. J. Fluid Mech. 688, 422-442.

LaCasce, J. H. \& BRInk, K. H. 2000 Geostrophic turbulence over a slope. J. Phys. Oceanogr. 30 (6), 1305-1324.

Pedlosky, J. 1987 Geophysical Fluid Dynamics, 2nd edn. Springer.

QIU, X.-L., XIA, K.-Q. \& ToNG, P. 2005 Experimental study of velocity boundary layer near a rough conducting surface in turbulent natural convection. J. Turbul. 6, N30.

Rajaei, H., Joshi, P., Alards, K. M. J., Kunnen, R. P. J., Toschi, F. \& Clercx, H. J. H. 2016 Transitions in turbulent rotating convection: a Lagrangian perspective. Phys. Rev. E 93 (4), 043129.

Rossby, H. T. 1969 A study of Bénard convection with and without rotation. J. Fluid Mech. 36, 309-335.

Shen, Y., Tong, P. \& XiA, K.-Q. 1996 Turbulent convection over rough surfaces. Phys. Rev. Lett. 76 (6), 908-911.

Stevens, R. J. A. M., Clercx, H. J. H. \& Lohse, D. 2010 Optimal Prandtl number for heat transfer in rotating Rayleigh-Bénard convection. New J. Phys. 12 (7), 075005.

Stevens, R. J. A. M., ClercX, H. J. H. \& Lohse, D. $2013 a$ Heat transport and flow structure in rotating Rayleigh-Bénard convection. Eur. J. Mech. (B/Fluids) 40, 41-49.

Stevens, R. J. A. M., van Der Poel, E. P., Grossmann, S. \& Lohse, D. $2013 b$ The unifying theory of scaling in thermal convection: the updated prefactors. J. Fluid Mech. 730, 295-308.

Stevens, R. J. A. M., Zhong, J.-Q., Clercx, H. J. H., Ahlers, G. \& Lohse, D. 2009 Transitions between turbulent states in rotating Rayleigh-Bénard convection. Phys. Rev. Lett. 103 (2), 024503.

Stringano, G., PAscazio, G. \& Verzicco, R. 2006 Turbulent thermal convection over grooved plates. J. Fluid Mech. 557, 307-336.

Tisserand, J.-C., Creyssels, M., Gasteuil, Y., Pabiou, H., Gibert, M., Castaing, B. \& ChIlla, F. 2011 Comparison between rough and smooth plates within the same RayleighBénard cell. Phys. Fluids 23 (1), 015105. 


\section{P. Joshi, H. Rajaei, R. P. J. Kunnen and H. J. H. Clercx}

Toppaladoddi, S., Succi, S. \& Wettlaufer, J. S. 2017 Roughness as a route to the ultimate regime of thermal convection. Phys. Rev. Lett. 118, 074503.

VorobiefF, P. \& ECKe, R. E. 2002 Turbulent rotating convection: an experimental study. J. Fluid Mech. 458, 191-218.

WAGNeR, S. \& SHIShKinA, O. 2015 Heat flux enhancement by regular surface roughness in turbulent thermal convection. J. Fluid Mech. 763, 109-135.

Wei, P., Chan, T.-S., Ni, R., ZhaO, X.-Z. \& XiA, K.-Q. 2014 Heat transport properties of plates with smooth and rough surfaces in turbulent thermal convection. J. Fluid Mech. 740, 28-46.

Weiss, S., Stevens, R. J. A. M., Zhong, J.-Q., Clercx, H. J. H., Lohse, D. \& Ahlers, G. 2010 Finite-size effects lead to supercritical bifurcations in turbulent rotating Rayleigh-Bénard convection. Phys. Rev. Lett. 105 (22), 224501.

XI, H.-D., LAM, S. \& XIA, K.-Q. 2004 From laminar plumes to organized flows: the onset of large-scale circulation in turbulent thermal convection. J. Fluid Mech. 503, 47-56.

XIE, Y.-C.\& XIA, K.-Q. 2017 Turbulent thermal convection over rough plates with varying roughness geometries. arXiv:1703.03137.

ZHONG, J.-Q. \& AHLERS, G. 2010 Heat transport and the large-scale circulation in rotating turbulent Rayleigh-Bénard convection. J. Fluid Mech. 665, 300-333. 